\title{
Psychanalyse et transidentités : hétérotopies
}

\section{Psychoanalysis and Transidentities: Heterotopies.}

Thamy Ayouch

Maître de Conférence en psychologie clinique à l'Université Lille 3,

Professor Visitante Estrangeiro à l'Universidade de São Paulo

Psychanalyste, psychologue clinicien

18, avenue du Président Hoover, 59000, Lille

0611345026

thamy.ayouch@gmail.com 


\section{Résumés et mots-clés}

\section{Résumé}

\section{Objectifs}

Repenser la filiation psychiatrique de nombre de théorisations analytiques de la « transsexualité », en désigner les points de butée, pour voir si et comment il est possible de penser une psychanalyse de la post-transsexualité.

Chercher à voir si la théorisation psychanalytique peut se départir de certains dogmatismes théoriques et recouvrer sa visée hétérotopique.

\section{Méthode}

Mise en exergue de l'hétérotopie comme motif commun aux transidentités et à la psychanalyse. Qu'elles présentent un mimétisme de la binarité de genre, ou une conception transgenre bouleversant cette binarité, les transidentités produisent des hétérotopies des modèles du féminin et du masculin. L'approche psychanalytique vise, elle aussi, dans son fonctionnement, une dimension hétérotopique : elle articule un paradoxal "savoir de l'inconscient», où le savoir et ses catégories positives sont déconstruits, dans un questionnement de l'origine et de l'adresse de toute posture discursive, et suit, elle aussi, les six principes foucaldiens de l'hétérotopie.

Mise en exergue de l'historicité de catégories de la métapsychologie, perpétrant une préoccupante maltraitance théorique, clinique et idéologique, et procédant de visions essentialisées des sexes et des genres. La fluidité de la théorisation analytique se rigidifie dans certaines théorisations des « transsexualités », les rapprochant de la psychose ou de la perversion, et les inscrivant dans un refus de la différence des sexes.

\section{$\underline{\text { Résultats }}$}

Déconstruction de certains outils analytiques ou psychiatriques pour repenser les transidentités.

Les réaménagements sont théoriques: il s'agit de penser une psychanalyse hétérotopique, foucaldienne et ouverte aux apports féconds des Gender, Queer et Transgender Studies. Il s'agit également d'aborder par la multiplicité les apories de la binarité ou de l'identité.

Les réaménagements sont également cliniques et articulent une tentative de repenser les protocoles, de pointer la maltraitance des visées «thérapeutiques» et de restituer l'expertise aux sujets trans.

\section{Discussion}


Comment concilier la pertinence d'instruments théoriques et leur véridiction (leur archéologie et leur généalogie), pour les resignifier? Le propos est de concevoir des instruments métapsychologiques et cliniques susceptibles de rendre compte de la spécificité des identifications et des vécus transidentitaires, et construits par delà la normativité sociale, culturelle et politique de la binarité des sexes.

\title{
Conclusions
}

Nécessité pour une approche psychanalytique d'écouter les savoirs locaux et minoritaires.

Nécessité pour cette approche de se détacher des visées psychiatriques d'évaluation de la « transsexualité » et du « vrai sexe ».

Nécessité de réaffirmer l'hypersingularité de chaque sujet trans par delà la généralité de la nosographie ou des catégories étiologiques.

Nécessité de repenser les contre-transferts théoriques et cliniques induits par ces questions et d'historiciser la catégorie de « différence des sexes ».

\section{Mots-clés}

Transidentités, transsexualisme, psychanalyse, genre, différence des sexes, hétérotopies, Foucault

\begin{abstract}
Objectives

The aim of this article is to set forth the psychiatric origin of psychoanalytical theories of "transsexuality", point out their limits, and ponder over the possibility of a posttranssexuality psychoanalysis.

The article asks whether psychoanalytical theory can move away from theoretical dogmatism and recover its heterotopic objective.
\end{abstract}

\section{Method}

The article starts setting forth heterotopy as a motive common to transidentities and psychoanalysis. Transidentities, either by mimicking or upsetting gender binarity, always conjure up heterotopies of femininity and masculinity. Psychoanalysis' approach too aims towards heterotopy: it sets forward a paradoxical "knowledge of the unconscious" that deconstructs positive knowledge categories and questions the origin and address of any discursive position. In this respect, it follows the six foucaldian characteristics of heterotopy. 
The text later dwells on the historicity of certain metapsychology categories which prove to be theoretically and clinically abusive and proceed from essencialized conceptions of sexes and genders. Psychoanalysis' fluid perspective may stiffen up in some theories of "transsexuality" that assimilate it to psychosis or perversion, or to a denial of sexual difference.

\section{$\underline{\text { Results }}$}

Certain psychoanalytical and psychiatric tools to tackle transidentities are deconstructed.

Theory is reorganized: the aim is to examine the possibility of a heterotopic psychoanalysis, that turns out to be foucaldian and open to the fertile perspective of Gender, Queer and Transgender Studies. The article also tries to use multiplicity to go beyond the aporiae of binarity and identity.

Clinical results consist in questioning official protocols, pointing out the ill-treating "therapeutic" perspectives and restoring expertise to trans subjects.

\section{Discussion}

How to reconcile the relevance of theoretical tools and their veridiction (their archaeology and genealogy), in order to resignify them? The article tries to conceive of metapsychological and clinical tools likely to account for specific transidentitary identifications and experience, and move beyond the social, cultural and political normativity of sex binarity.

\section{Conclusions}

Necessity for a psychoanalytical approach to listen to local and minority knowledges.

Necessity to move away from psychiatric assessment of "transexuality" and the "real sex".

Necessity to reassert every trans subject's hypersingularity, beyond the general nosographies and etiological categories.

Necessity to think over the theoretical and clinical counter-transferences produced by these questions, and historicize the category of "sexual difference".

\section{Key-words}

Transidentities, transsexuality, psychoanalysis, gender, sexual difference, heterotopies, Foucault 


\section{Psychanalyse et transidentités : hétérotopies}

La «transsexualité » est une catégorie née de la rencontre du médical et du juridique. Si la psychanalyse, dans sa rupture épistémologique, n'a pas vocation à reprendre le savoir psychiatrique, bon nombre de discours analytiques sont soucieux de voir déjà chez Freud les fondements classificatoires d'une pathologie «transsexuelle», et se développent alors dans deux directions : un freudisme nord-américain médicalisé, et un lacanisme fustigeant les errances de la médecine.

Plus ici qu'ailleurs, la psychanalyse n'échappe pas aux formations discursives de son époque, et s'inscrit dans la droite lignée de la psychiatrie. Cet oubli de son historicisation ne se paie-t-il pas toutefois d'un abandon de sa dimension proprement psychanalytique? Depuis Freud, la pensée psychanalytique s'applique à étudier la rencontre, dans toute théorisation, des logiques de la connaissance et du désir, et à pointer la menace de fétichisation et de dogmatisation qui guette toute théorie. Révélant le sous-bassement pulsionnel de toute connaissance et critiquant les implications métaphysiques, voire psychopathologiques de la théorisation, la psychanalyse ne saurait exempter de cette critique ses propres constructions théoriques. Faire taire ce questionnement ne revient-il pas à abandonner ce pouvoir de mettre en crise, qui lui est propre, à la faveur d'une fureur classificatrice et normative? A considérer la « transsexualité » comme catégorie existante, ontologiquement, biologiquement ou symboliquement déterminée, la psychanalyse ne devient-elle pas anti-psychanalytique, et ne produit-elle pas ce que Derrida nommait une « résistance auto-immunitaire de la psychanalyse à son dehors comme à elle-même » [1] ?

Mon propos sera de tenter de penser cette filiation psychiatrique de nombre de théorisations analytiques de la «transsexualité », d'en désigner les points de butée, pour voir si et comment il est possible de penser une psychanalyse de la post-transsexualité. Mais au préalable, il est pertinent de se pencher sur le motif de l'hétérotopie, qui semble rapprocher psychanalyse et transidentités eu égard à leur subversion constante de l'identité.

\section{Le motif hétérotopique}

Le terme parapluie «transidentités », forgé par la sociologue Heike Boedeker, désigne les identifications en marge de la binarité des catégories de sexe, genre et sexualité, et renvoie à une multiplicité d'expériences (intersexes, travestis, hommes féminins et femmes masculines, «transsexuels » de la psychiatrie, transgenres, et personnes en 
questionnement sur la pertinence des catégories de sexe) [2]. Les transidentités présentent, nous semble-t-il, des hétérotopies des modèles du féminin et du masculin, des délocalisations et des transformations des sexuations. Contre-espace pensé par Foucault, l'hétérotopie accueille, comme le bordel, le jardin ou le bateau, les marges et les déviations, à la fois internes et externes, et juxtapose plusieurs espaces, plusieurs temps et plusieurs modèles. Les transidentités semblent ainsi suivre les six principes établis par Foucault sur les hétérotopies [3]: elles sont universelles, ont une fonction particulière dans chaque culture, juxtaposent plusieurs principes incompatibles dans la norme de genre, introduisent des hétérochronies, sont reliées à la norme dans un système d'ouverture et de fermeture, et créent un espace d'illusion qui dénonce comme encore plus illusoire la performance de genre.

L'approche psychanalytique elle aussi vise, dans son fonctionnement, une dimension hétérotopique : elle articule un paradoxal «savoir de l'inconscient», où le savoir même et ses catégories positives sont déconstruits. La rupture épistémologique du discours analytique consiste à pointer la gageure et les limites de toute procédure cognitive et son infiltration par des enjeux autres que ceux du savoir : des visées pulsionnelles sur le plan subjectif, un dispositif de pouvoirs sur le plan collectif. La signifiance d'un discours ne tient pas aux divers sens positifs articulés par ses contenus, mais à son origine et son adresse. De cette analyse de l'énonciation plutôt que de l'énoncé, la théorisation, pour rester psychanalytique, ne saurait s'exempter.

C'est pourquoi, sans toutefois être forme-sens (qui ferait d'elle un ensemble d'hermétiques vaticinations) la théorisation psychanalytique aspire ainsi à emprunter à l'inconscient sa dimension hétérotopique, à nouveau selon les six principes foucaldiens de l'hétérotopie: elle se veut à même d'accueillir l'inattendu qu'il lui réserve, dans toutes les configurations historiques et dans ses fonctions particulières au sein de chaque culture, juxtapose plusieurs modes d'explicitation incompatibles dans un seul modèle scientifique (mythe heuristique, thermodynamique, pseudo-biologie, linguistique, topologie), introduit de l'hétérochronie par l'intempestivité de ses remises en question et par son historicisation, reste reliée à la norme par un système de fermeture et d'ouverture et crée un espace d'illusion rendant encore plus illusoire le fonctionnement de toute théorie et son aspiration au savoir-pouvoir.

C'est, semble-t-il, ce système d'ouverture/fermeture à la norme qui semble le plus garant $\mathrm{du}$ statut hétérotopique de la théorisation analytique, et qui se rigidifie dans la considération des transidentités. La perte de cette posture hétérotopique procède d'une filiation psychiatrique d'emblée pathologisante, qu'il semble pertinent d'aborder maintenant. 


\section{La fabrication psychiatrique}

L'invention de l'homosexualité et de la transsexualité sont conjointes : leurs destins sont liés dans l'agrégat psychiatrique de la notion de sexe, sexuation indissociable d'une sexualité. La médicalisation des perversions aux XIXè siècle procède au repérage classificatoire d'anomalies, qui, en retour, instituent une norme sexuelle, inscrite dans une configuration fixe du genre. Aux hommes et aux femmes reviennent par nature des activités génitales de commerce avec l' " autre sexe », où l'idéal reste de besogner sans trop de façons son épouse par coït membrum virile in vaginam, et tout le reste n'est que littérature... Littérature psychiatrique abondante, proliférante et classificatrice.

Introduit par Magnus Hirschfeld en 1910, le terme "transsexualisme » désigne un travestissement accompagné, pour les hommes, de la conviction d'être des femmes. C'est toutefois en 1951 que naît le "phénomène transsexuel », selon l'expression du sexologue Harry Benjamin, avec l'opération largement médiatisée de Christine Jorgensen. Se popularisent alors conjointement, aux Etats-Unis, un behaviorisme endocrinologique et une théorisation de filiation freudienne, représentée essentiellement par Robert Stoller.

La transsexualité n'advient donc, dans sa version moderne, que par la grâce d'une médecine qui la génère dans sa forme diagnostique. Sa reconnaissance, sur le triple plan social, médical et juridique apparaît indissociable de sa pathologisation: pour être identifié/e comme «transsexuel/le », et avoir ainsi accès, aujourd'hui, aux protocoles officiels de réassignation de genre, un diagnostic de pathologie mentale («transsexualisme », « trouble de l'identité de genre » ou " dysphorie de genre » selon les versions du DSM) doit être émis par une équipe officielle de psychiatres, psychologues et endocrinologues. Le changement juridique de la mention de "sexe », en France, reste alors conditionné à une chirurgie de réassignation ou à la stérilisation du sujet.

Le discours scientifique qui prétend ici articuler une vérité sur la «transsexualité 》 et procéder de l'épistémè, n'est toutefois qu'une opinion parmi d'autres sur ce phénomène fabriqué, et s'inscrit ainsi dans la doxa. Plus encore, comme le soulignait G. LantériLaura au sujet de la médicalisation des perversions, c'est ici la doxa qui délimite le champ de phénomènes dont l'épistémè traite: l'opinion circonscrit la pathologie transsexuelle dans le cadre du dimorphisme de genre qu'elle vient contrarier, et la production de savoirs scientifiques reste tributaire de cette opinion.

C'est alors cette toute doxique épistémè que semblent reprendre bon nombre de théorisations psychanalytiques de la transsexualité. 


\section{Théorisations psychanalytiques}

La lecture de l'abondante littérature analytique sur «le transsexualisme » révèle une navrante indigence en créativité théorique, et une préoccupante surdité clinique. Selon qu'ils se déclarent «freudiens» ou « lacaniens », les auteurs instituent un accès à la réassignation réglementé, codifié et conditionné par des gages de conformité de genre, ou condamnent la réassignation de sexe considérée comme réponse folle à une demande folle.

Pour Robert Stoller [4] l'identité de genre, distincte du sexe, et organisée tout au long d'une vie, reposerait sur la conviction d'être masculin ou féminin. Si cet auteur a le mérite d'introduire, en 1968, la catégorie de genre, il la réduit toutefois une construction sociale advenant seulement dans un deuxième temps sur la base du substrat biologique du sexe, ontologiquement et irréductiblement premier. L'ancienneté de la conviction d'être de l' « autre » genre déciderait d'un départ entre «transsexuels primaires », et «transsexuels secondaires ». Dans sa description étiologique des trois générations nécessaires pour produire un/e « transsexuel/le », la tradition psychiatrique dégénérative du XIXè siècle le dispute à l'impérissable familialisme que Deleuze et Guattari prêtaient à la psychanalyse.

Colette Chiland $[5,6]$, son héritière française, conçoit le "transsexualisme » comme « maladie du narcissisme », construction du self par « identité du sexe contraire » [7], et les personnes trans comme sujets états-limites pour qui toute élaboration est courtcircuitée, évacuée dans l'acte et le corps [5,7]. Le dimorphisme est explicitement revendiqué au nom de l'évidence biologique de la « boussole du sexe » [7].

Si les «freudiens » cherchent à débusquer les «vrais transsexuels» (primaires), pour permettre l'accès protocolisé aux soins, pour la veine lacanienne, ce forçage du Réel par la chirurgie est psychotique : l'erreur, comme le soutient Lacan [8], consiste à prendre l'organe (pénis) pour le signifiant (phallus). Le discours de bien des analystes lacaniens reste à l'avenant : ainsi M. Safouan inscrit-il la «transsexualité » dans une «forclusion du Nom-du-Père » [9] entraînant la confusion du phallus et du pénis ${ }^{1}$. Cette même analyse est reprise par J. Dor $[10]^{2}$, M. Czermak [11] ${ }^{3}$, H. Frignet $[13]^{4}$, ou G. Morel

\footnotetext{
${ }^{1}$ Selon M. Safouan, le sujet «transsexuel» ne reconnaît pas le grand Autre, enlisé dans un commerce avec le seul « autre de la demande, sans l'au-delà obscur du désir » [9]. Ce qui est nié dans le symbolique revient dans le réel : répondant à la demande, le sujet transsexuel recourt à la chirurgie, qui rend alors effective sa castration - la castration symbolique lui étant impossible. L'erreur du sujet transsexuel consiste à demander une ablation du pénis pour se libérer du phallus.

${ }^{2}$ Pour Joël Dor également, le/la «transsexuel/le », à la frontière entre psychose et perversion, confondant l'organe et le signifiant, n'accèderait qu'à une castration réelle, chirurgicale. Son identité sexuelle serait alors impossible, faute d'un accès au statut symbolique de la différence des sexes. Si les « hommes» (entendons ici les femmes trans, MtF) sont du côté de la nécessité phallique de l'être, et « cette assignation
} 
Perpétrant une préoccupante maltraitance théorique, clinique et idéologique ${ }^{6}$, ces métapsychologies procèdent de visions essentialisées des sexes et des genres, répètent les rigidités d'une masculinité et d'une féminité supposées fondées, de manière évidente, dans le biologique ou le symbolique, et instituent une conception anhistorique du phallus, de la jouissance, des sexuations et des sexualités.

Cette étrange crispation, et l'interdit de pensée ici inlassablement répété tiennent, semble-t-il, à l'ampleur du contre-transfert clinique et théorique mobilisé. L'hostilité de ces praticiens semble renvoyer à l'inquiétante étrangeté ici éprouvée, mélange d'inquiétude, d'effroi, de bouleversement et de fascination que le motif transidentitaire peut provoquer en chacun/e.

irréversible les condamne, peu ou prou, à assumer une immigration quasi inévitable dans le ghetto des psychoses » ([10], p 255), les « femmes transsexuelles » (ou plutôt hommes trans, FtM) se situent plus du côté de la nécessité phallique de l'avoir, communément à l'œuvre dans les perversions.

${ }^{3}$ De même, pour M. Czermak, le/la transsexuel/le montrerait la pathologie de l'identité sexuelle existant dans toute organisation psychotique/ : il/elle confondrait le Phallus symbolique avec un pénis réel, et prendrait ainsi l'organe pour le signifiant. Cet auteur reconnaît au transsexualisme le mérite d'amener au premier plan un réel, «c'est à dire ce que la psychanalyse entend comme l'impossible : impossible en effet de ne pas être ou un homme ou une femme ». Ici, «l'identification sexuelle» est appartenance, qui « ne tient son origine que de l'altérité réelle qui procède de l'organisation sexuée de l'espèce humaine : il faut nécessairement la conjonction de deux éléments appartenant à deux ensembles disjoints pour qu'il y ait reproduction » ([11], p 15).

${ }^{4}$ Dans cette même lignée, toute remise en question de la binarité de genre par les transsexualistes est interprétée, par H. Frignet, comme tentative d'échapper à la castration en substituant à un manque symbolique une coupure dans le réel. Cette absence de reconnaissance du phallus symbolique placerait le sujet transsexuel hors du sexe [12].

${ }^{5}$ Selon G. Morel, «le transsexualiste » refuse d'inscrire sa jouissance dans la fonction phallique et, psychotique, se passe du Nom-du-père. «Il « « est celui qui veut se libérer de l'erreur qui a fait passer au réel, par le biais du langage, la petite différence anatomique. Il veut donc changer d'organe pour se libérer de cette erreur, puisque c'est à partir de l'organe qu'il a été signifié garçon ou fille dans les catégories phalliques pour lui forcloses. En fait, c'est une folie, car ce n'est pas l'organe, mais le signifiant qu'il rejette, comme signifiant de la jouissance sexuelle qui pour lui est trop réelle, de ne pas s'être corrélée au phallus » ([13], p 199).

${ }^{6}$ La maltraitance tient ici à l'utilisation injurieuse de catégories «diagnostiques ». Si, dans certaines métapsychologies, la catégorie de "psychose» ne renvoie qu'à une structuration, une intendance du matériel signifiant inconscient, distincte de la névrose, ce n'est pas le cas ici. Cette catégorie n'est nullement anodine ou «neutre » : elle a une fonction politique, produit des effets de pouvoir et sert dès l'origine à mettre en évidence la dimension de «demande folle», de délire sur l' «identité », et à psychiatriser les personnes trans consultant (les rencontres de patients trans par Lacan ou Czermak [12], par exemple en attestent, et le propos reste, dans cet article, de mettre en exergue la filiation psychiatrique de certaines conceptions analytiques). En résulte une pathologisation, du reste propre au protocole psychiatrique. En outre, il convient de se pencher sur la charge fantasmatique charriée par les termes de «psychose » ou «perversion » qui, bien qu'ils ne prétendent parfois faire référence qu'à une organisation et au mode de formation du symptôme, servent ici d' " injure diagnostique ». Dans ces métapsychologies, l'injure consiste à subsumer l'individu trans, quel qu'il soit, sous la catégorie nosographique générale de la psychose ou de la perversion, qui aurait raison de toute sa singularité. En outre, l'injure réalise ici une performativité : la structure psychotique ou perverse appliquée aux personnes trans produit alors une subjectivité générale, qui toutefois ne précède pas sa nomination. Cette performativité est dissimulée par la référence à un espace pré-diagnostique, psychique, universel, de la psychose ou de la perversion transsexuelle, ici prétendument révélé par ces analyses. 
La théorisation psychanalytique peut-elle alors se départir de cette ontologie du genre qui la traverse, et recouvrer sa visée hétérotopique ?

\section{Hétérotopies théoriques}

L'hétérotopie théorique ou clinique de la psychanalyse tient, nous l'avons vu, à sa capacité d'accueillir l'inattendu, à sa désacralisation de tout modèle d'intelligibilité, « échafaudage » qu'elle ne prend pas " pour le bâtiment lui-même » [15] et dont elle indique, par sa métaphorisation théorique, la performativité. Son hétérochronie procède de son ambition à échapper aux formations discursives qui lui sont contemporaines. Il s'agit alors de relire les topoi théoriques et cliniques dogmatisés, et de concevoir, au sein de la théorie analytique, des possibilités non mises en exergue et rappelées par les visées fondamentales de l'écoute clinique. Cette hétérotopie de la psychanalyse relève également de sa capacité d'hybridation par d'autres approches, de son accueil du discours foucaldien et des analyses des Gender, Queer et Transgender Studies.

\section{Une psychanalyse foucaldienne}

Comme le notait Foucault au sujet de la notion de «vrai sexe» [16], dans la « transsexualité », la vérité du sujet se trouve réduite à son identité sexuelle : le sexe est soumis à un régime de véridiction. Une psychanalyse susceptible d'accueillir les transidentités ne peut s'épargner d'intégrer l'analyse foucaldienne du «dispositif de sexualité » [17] à sa théorisation. Soucieuse de faire l'archéologie de la catégorie de sexe (sexuation, sexualité, sexuel-infantile) dans son propre discours, elle pourrait reprendre la critique foucaldienne du sexe-désir ([17], p 208). Le sexe-désir présente le sexe (appareil génital) comme cause du désir, et fait du désir la conséquence du sexe, dans un trajet normatif qui va de la sexuation d'hommes-pénis et femmes-vagins à une hétérosexualité obligatoire. De ce sexe-désir, liant de manière univoque sexuation et sexualité, semble découler la catégorie psychanalytique de « différence des sexes », qui reprend, sans distance aucune, une essentialisation dimorphiste, comme nous allons le voir plus avant. L'usage du terme «transsexuel» perpétue les normes de genre qui imposent une dualité des sexes et leur manifestation métonymique par les organes génitaux, et condamne la psychanalyse à ne pas s'affranchir de ce dispositif de sexualité.

Une psychanalyse « foucaldienne » pourrait tenter, en outre, d'inscrire la sexuation dans une subjectivation conçue non plus selon l'hypothèse répressive du pouvoir juridicomonarchique, mais selon la réalité du pouvoir disciplinaire-stratégique [16]. L'hypothèse répressive que Foucault attribue à une psychanalyse schématisant le 
pouvoir sous la forme du père séparateur ou législateur semble advenir au moment précisément où la loi patriarcale, propre au système juridique-monarchique, est remplacée par le jeu stratégique de pouvoirs propre au système disciplinaire. Il s'agirait de penser la structuration psychique plus que par la Loi, définie dans le cadre d'un Symbolique articulant l'Edipe, le Nom-du-Père ou le Phallus comme catégories immuables, par la norme, que les transidentités viennent subvertir tout en la répétant. Selon Foucault, le régime de la loi ne permet pas de saisir le véritable rapport du désir au pouvoir, qui ne fonctionne pas en interdisant seulement. Que serait alors une métapsychologie articulée en termes de pouvoir et résistance, et non fondée sur le négatif, la loi, et la centralité de la fonction paternelle ? Nous tenterons d'esquisser ici quelques pistes.

Pour Foucault, le pouvoir désigne un ensemble de relations entre "partenaires » : c'est une conduite de la conduite, une gouvernementalité, procédant de stratégies, qui mêlent pouvoir et résistance [18]. Au cœur des relations de pouvoir se trouvent des libertés rétives, des retournements, des stratégies de lutte, limites et conditions de ces stratégies de pouvoir. C'est probablement cette question d'un sujet psychique subjectivé par le pouvoir qu'aborde Judith Butler [19] en tentant de tenir ensemble pouvoir et psyché à travers le concept d'assujettissement. L'assujettissement est pensé en référence à un attachement passionné du sujet à ceux dont il dépend originairement de manière fondamentale. Butler permet alors de penser le sujet à la fois comme effet d'un assujettissement par soumission au pouvoir et lieu d'une subjectivation par retournement de ce pouvoir sur soi.

Par ce pouvoir disciplinaire de la norme agissant sur le sujet et le faisant agir, il devient possible de penser psychanalytiquement sans les pathologiser les configurations de genre, de sexe et de sexualité qui viennent contester le primat d'une conception binaire de la différence des sexes. Le choix inconscient d'une sexuation n'est pas alors une manière de se retrancher du régime, seulement négatif, de la Loi et de l'interdit. Ce n'est pas ici un refus de la «boussole du sexe», ni de la congruence «naturelle» du sexe « biologique » supposément premier et de l'identité de genre, qui provoquerait un courtcircuitage du psychique [7] par passage à l'acte (transformation du corps). Ce n'est pas non-plus une forclusion du Nom-du-Père ou un refus du Phallus comme signifiant maître, bannissant le sujet dans l'espace hors-sexe de la non-symbolisation (structure psychotique). Ce serait plutôt une identification de genre advenant dans un jeu de forces et de libertés rétives assujettissant et subjectivant à la fois. Conçue dans le cadre de jeux de pouvoir et de résistance aux normes de genre, l'identification de genre apparaît comme "pratique d'improvisation qui se déploie à l'intérieur d'une scène de contrainte »[20]. Si l'assujettissement au normes est constant, les modes de 
subjectivation sont susceptibles de se transformer : les normes peuvent être reprises et contestées, par une résistance qui surgit de l'intérieur des relations de pouvoir. Et la psychanalyse, comme théorie et clinique, se doit d'ouvrir un espace pour ces reconfigurations : il lui revient, en rappelant l'historicité des normes, d'entendre la subjectivation par delà les catégories prétendument universelles et anhistoriques de la Loi (« différence des sexes », « Phallus », « Noms-du-Père », etc.).

\section{Du binaire au multiple}

Il convient donc de se défaire de la notion psychiatrique de « transsexualité », tributaire du primat d'une binarité de la différence des sexes. Plus qu'une quelconque évidence naturelle, celle-ci est une production du système sexe-genre, une relation de pouvoir inscrite dans d'autres relations de pouvoir, qui construit les catégories de « sexe» ou de « roc biologique » comme ontologiquement antérieures à l'espace culturel et discursif qui les produit.

Accueillir la possibilité transgenre, qui délie la sexuation de l'appareil génital, signifie également renoncer à l'idée, problématique, d'une sexualité «homosexuelle» ou « hétérosexuelle» comme « choix du même » ou de l' «autre ». Dans le désir trans, la sexualité n'est pas liée à la stabilité ou à l'existence des sexes. Une personne transgenre peut être attirée par des hommes, des femmes, ou «the rest of us » [21], sans qu'il soit possible de dire qu'elle cherche «son» sexe ou l' «autre». La multiplicité des combinaisons et l'impossibilité d'un point de vue classificatoire objectif rejoignent ainsi le paradigme psychanalytique de la pulsion. Rappelons que la notion freudienne de sexualité infantile, perverse polymorphe, procédant de la pulsion, ne manque pas d'introduire une inédite dé-naturalisation de la sexualité, mais aussi de la sexuation. Lorsque la visée de la sexualité n'est pas le destin biologique de la reproduction, la complémentarité de deux sexes de l'espèce apparaît toute relative. Plus encore, c'est essentiellement leur définition qui semble problématique. Freud souligne en effet à plusieurs reprises que si les notions de «masculin» et de «féminin» sont "peu équivoques pour l'opinion commune », elles restent de part en part « confuses » [22]. La psychanalyse «ne peut (...) élucider» l'essence de ces notions, « elle reprend à son compte les concepts et les met à la base des ses travaux $»^{7}$. Comme il le souligne dans un autre texte, «le contenu des constructions théoriques de la masculinité pure et de la féminité pure reste incertain » ([24], p 132), et les répartitions du masculin et de féminin ne procèdent pas d'une séparation, mais d'un chevauchement, d'un entrecroisement au sein du même sexe/genre, d'« un vacillement dans les opérations visant à statuer sur la

\footnotetext{
${ }^{7}$ « Si l'on tente de les ramener à des principes plus originaires, la masculinité se volatilise en activité, et la féminité en passivité, ce qui est trop peu », [23], p 270.
} 
différence » [25].

Davantage différence de sexe ${ }^{8}$ que des sexes, cette différence doit être conçue hors de l'exclusion d'un genre par 1' (les) autre(s), par delà la révolte, « masculine », contre l'attitude passive à l'endroit d'un homme, et l'envie, « féminine », du pénis thématisées par Freud [27]. Une déconstruction psychanalytique en est proposée par Jean Laplanche, qui conçoit le sexual (propre à l'inconscient) comme résidu inconscient du refoulementsymbolisation du genre, pluriel, par le sexe, duel [26]. Dans l'opération de traduction des signifiants énigmatiques transmis de l'adulte à l'enfant, le sexe, dualisé par la reproduction sexuée ou la symbolisation en présence/absence, phallique/castré, vient organiser la pluralité du genre qui le précède. Si les normes sont ici duelles, les possibilités psychiques sont multiples, et l'inconscient, comme restes de la traduction de ces messages énigmatiques, recèle cette multiplicité.

Gageons alors que lorsqu'elle ontologise la «différence des sexes », la théorie psychanalytique devient théorie sexuelle infantile de son auteur. L'interprétation de la sexuation comme possession ou privation du pénis est empreinte de l'imaginaire historique, et partant, subjectif, dans lequel elle advient : elle reste historiquement située dans une perspective phallogocentrique qu'on souhaiterait différente actuellement.

La « différence des sexes »n'existe pas à l'état pur, ni au titre d'un constat tributaire d'un marqueur unique, ni à celui d'une indépassable binarité des jouissances «phallique» et «pas-toute» [28]. Le sexe biologique renvoie à une multitude d'indicateurs susceptibles de degrés : l'unification de ces données hétérogènes et leur réduction à une seule est un acte social, produisant une perception-interprétation de la différence de deux appareils génitaux. En outre, si les formules de la sexuation lacaniennes entendent définir des postures masculine et féminine quant au rapport au phallus et à la jouissance et abandonnent la référence à un sexe défini biologiquement, elles n'en invoquent pas moins, pour être construites, deux groupes universels : « les hommes ", « les femmes ", et un au-moins-un déjà sexué, le père de la horde primitive. S'il n'est plus question de femmes et hommes définis biologiquement ou socialement, mais d'incommensurables types de jouissance ("côté homme», «côté femme») qui peuvent revenir tout autant à des hommes qu'à des femmes, à la base de leur définition logique se trouvent deux côtés («côté homme», "côté femme»), fondés sur deux groupes convoquant, pour être constitués, une différence des sexes naturalisée et binaire. De même, il semble judicieux d'interroger l'inscription historique du féminin comme tout-Autre (situant l'hétérosexualité non plus du côté de la fonction paternelle, mais de

\footnotetext{
${ }^{8}$ «Pour l'être humain, la perception des organes génitaux n'est plus la perception de deux organes génitaux mais d'un seul. La différence des sexes devient 'différence de sexe' », [26, p 171].
} 
l'ouverture à une jouissance féminine), et de questionner le point de vue (masculin, hétérocentré) à partir duquel est présentée cette catégorie 9 .

Si donc la différence des sexes n'est pas une catégorie physique ou symbolique isolable des actes sociaux par lesquels nous la constituons en réalité pertinente et visible de nos pratiques et de nos désirs, l'inscription dans l'une ou l'autre des catégories de sexe instituées, et la subjectivation-assujettissement qui en découle pour l'enfant ou l'adulte dépendent de stratégies de pouvoirs et de résistances. Et la contestation trans ou queer de ces catégories est susceptible d'ouvrir un nouvel espace de subjectivationassujettissement à la croisée de ces relations de pouvoir : elle n'est pas le fait d'une pathologie psychique. Concevoir la subjectivation comme assujettissement passionné permet de rendre compte de la spécificité des identifications et des vécus transidentitaires, au plus proches de la singularité de ces expériences, et par delà la normativité sociale, culturelle et politique de la binarité des sexes au fondement de la théorisation.

En outre, plus que le deux de la «différence des sexes» et de la «bisexualité psychique », c'est également l'Un de l'identité qu'il semble pertinent de déconstruire ici, pour repenser les identifications de genre dans la multiplicité qui les caractérise. Si la matérialité des corps et leurs dissemblances renvoient à une multiplicité de données biologiques et symboliques, l'unification de celles-ci en un marqueur unique du sexe est un processus imaginaire. Une psychanalyse hétérotopique ne peut manquer d'analyser ce processus d'unité fantasmatique, et son rejet de la multiplicité, de la fragmentation et de l'indétermination propres à la théorie des pulsions. Cette unification se révèle défensive contre le mouvement, l'ambivalence, et le conflit caractérisant l'inconscient. Il s'agit donc, tant en psychanalyse que dans les Gender et Queer Studies, de considérer que l' «identité » sexuée et sexuelle n'est jamais fixe, mais correspond à une posture défensive, imaginaire, qui fomente, autant chez des personnes trans que cis $^{10}$, l'illusion narcissique d'une «essence» masculine ou féminine, hétéro ou homosexuelle. Les

\footnotetext{
${ }^{9}$ Le propos est de distinguer ici d'une part certains développements du Séminaire $X X$ (la rupture avec la logique ensembliste, permettant d'avancer que la femme, soumise à la catégorie "pas-toute », n'existe pas, ou l'incommensurabilité de la jouissance pas-toute illimitée, à la jouissance phallique), et, d'autre part, leurs conditions de possibilité d'un point de vue archéologique et généalogique : le recours au trope, situé, du féminin comme tout-Autre, et à deux catégories "côté homme » et côté femme », tout autant historiquement définies. Convoquer ces deux groupes ici revient à placer au fondement du raisonnement une binarité non questionnée historiquement (bien qu'à l'évidence, la jouissance pas-toute en arrive à subvertir cette binarité). Concevoir toutefois ces notions comme anhistoriques permet de faire l'économie d'une analyse de l'homophobie et de la transphobie qui guettent les théories analytiques et peut entraîner de lourdes conséquences cliniques.

${ }^{10}$ Cis renvoie aux sujets qui ne sont pas dans un processus conscient de transition de genre (personnes non trans).
} 
formations de l'inconscient rompent toutefois avec ce fantasme de mêmeté et d'ipséité, et l'approche analytique préfère à l'identité la plasticité psychique des mouvements identificatoires, inchoatifs et inachevés.

\section{Hétérotopies cliniques}

\section{Repenser les protocoles}

L'invention récente du «transsexualisme» pointe l'historicité de cette catégorie, qui semble aujourd'hui bien peu représentative des configurations transidentitaires. Si les premières demandes de transgénitalisation adressées au milieu médical pouvaient s'inscrire dans la binarité de genre, aujourd'hui, la voie psychiatrique des protocoles ne répond plus, ni qualitativement ni quantitativement, aux demandes actuelles. Comme le note Arnaud Alessandrin [29], la scène juridico-médicale française est débordée par des demandes «hors cadres », où les corps trans innovent et inventent de nouvelles configurations des normes de genre.

La chirurgie de transgénitalisation apparait alors souvent comme une violence qui manque sa cible, et n'est souvent visée que pour permettre l'accès à un changement juridique de la mention de sexe dans les documents d'identité. Il s'agit donc de questionner la dimension «thérapeutique» dont se réclament les protocoles officiels soucieux de déterminer les « vrais transsexuels/les ». Il s'agit de se demander, comme le fait Karine Espineira, qui soigne ici, dans quel contexte et surtout, de quoi [30]. Ce «bouclier thérapeutique » [30], semble davantage protéger une armée d'experts de la « transsexualité » des contestations trans que «soigner» des patients «transsexuels» d'une souffrance d'identité de genre. La question est ici centrale eu égard au travail de l'analyste : il s'agit de se demander si sa visée est d'accompagner l'analysant/e dans son parcours psychique avec et contre les normes, ou de servir le maintien d'un ordre social. En outre, c'est la question même de la dimension «thérapeutique », seulement «bénéfice de surcroit » pour une approche analytique, qui est ici posée. Sous couvert de soigner, en les opérants, des individus qui présentent des identifications et des corps ne répondant pas aux normes de genre, une distinction entre « vrais » et « faux » trans est produite, formalisant une hiérarchie pyramidale entre catégories de trans (opéré/es, transgenres, travesti/es) [30]. Le dispositif opère, comme le souligne K. Espineira, un échange de bons procédés : un statut est aménagé pour les « vrais » trans, qui s’engagent à respecter l'ordre social des sexuations, des alliances et des filiations. Le «ici on soigne » propre à ce bouclier thérapeutique, revient à protéger des troubles dans le genre et identifications non binaires, et érige autour des « soignants» un mur " qui empêche, réfute et discrédite par avance toute avancée et tout dialogue » [30]. 
Pour éviter une clinique de la police des sexuations, l'approche analytique ne peut que se distinguer de toute thérapeutique : il convient de concevoir les transidentités non point comme exceptions de la subjectivation, mais possibilités réelles propres à chacun/e, et, partant, à l'analyste aussi, pour qui une constante analyse du contretransfert se révèle ici centrale.

Par delà le seul motif ipsocentriste d'une « souffrance subjective » détachée du contexte social, c'est une plus grande exposition à la vulnérabilité qui caractérise les identifications trans. La médecine, la psychologie ou la psychanalyse n'apparaissent pas tant comme modalités de traitement d'une souffrance transidentitaire qui précèderait leur intervention, mais comme procédures qui parfois semblent au contraire la créer, l'instituer, et contribuer à l'institutionnaliser. Face à cette vulnérabilité, l'approche psychanalytique doit se distinguer de toute injonction thérapeutique, qui annule d'entrée de jeu la position subjective. L'écoute psychanalytique n'a aucune visée thérapeutique : elle offre à l'analysant/e un espace de subjectivation, de désaliénation, et d'identification de son désir. Elle peut alors proposer d'accompagner dans leurs interrogations les sujets qui en formulent personnellement la demande, sans que le motif transidentitaire ne soit, d'emblée, placé au cœur de cet accompagnement.

\section{Expertise des sujets et déconstruction des maltraitances}

Une pratique clinique soucieuse de respecter l'hyper-singularité du sujet, et d'éviter de remplacer son désir par celui de l'analyste se doit alors d'être à l'écoute de la spécificité propre et non-généralisable de chaque analysant/e. Par delà toute construction théorique ou classification diagnostique rigides, elle cherchera à remettre les sujets concernés en position d'expertise, et à les faire participer directement à la reconfiguration de la «théorie psy» par laquelle ils sont pensés. Pour cela, il convient, comme y invite Françoise Sironi [31], de déconstruire, avec eux, les effets iatrogènes, délétères et toxiques de la prise en charge psychiatrique officielle de la « transsexualité ».

Il s'agit ici d'identifier les violents contre-transferts mobilisés dans les théories et pratiques cliniques qui ne peuvent accueillir sans la trahir l'expérience de vie des personnes trans. Cette transphobie professionnelle produit un lien thérapeutique paradoxal, mortifère, responsable d'une pathologie iatrogénique, réactionnelle. Il convient alors de considérer les «syndrome de persécution», «méfiance », « agressivité », « agitation », « dépression », « autodévalorisation », « tentatives de suicide ", attribués à tort à une prétendue " psychopathologie des sujets transsexuels » comme symptômes induits en grande partie par les théories et pratiques inadéquates. 
Apparaissent ainsi les situations paradoxales et l" « effort pour rendre fou » produits par les dispositifs cliniques et théoriques officiels, où les personnes trans sont soumises à une obligation de psychothérapie cherchant à la fois à vérifier la constance de leur demande et à les en dissuader. Si elles y renoncent, ce ne sont pas de «vrais transsexuels », si elles la maintiennent fermement, les voilà accusées de rigidité paranoïde. L'institution de la «transsexualité» produit ainsi de véritables contradictions : s'acharnant à soigner à tout prix une expérience identitaire pathologisée (la transidentité), les équipes négligent les troubles que provoquent leurs discriminations.

\section{Conclusion}

Les transidentités échappent à la production normative du sexe-genre, et étendent les limites de l'intelligibilité des corps. A l'institution d'une « souffrance transsexuelle » par l'expertise médicale font pendant les savoirs locaux ou minoritaires, délocalisés, des expériences transidentitaires. A charge alors à une écoute psychanalytique d'entendre ces délocalisations et l'hypersingularité de chaque construction transidentitaire, qui ne relève d'aucune « transsexualité » unitaire.

Pour ce faire, un certain nombre de réaménagements semble nécessaire ${ }^{11}$ :

- Il convient de se désolidariser des visées psychiatriques d'évaluation de la «transsexualité », de fabrication du «vrai sexe», et de maltraitance théorique, institutionnelle et clinique perpétrée à l'endroit des personnes trans.

- Il semble fondamental de réaffirmer la primauté de l'hyper-singularité de chaque sujet, par-delà toute fureur nosographique : mais d'un sujet inscrit dans son contexte socialhistorique. Un/e psychanalyste soucieux/se d'accompagner une personne trans, qui ne le/la consulte pas nécessairement au sujet de sa transition, ne peut prétendre "guérir " de la «transsexualité », et ne doit pas craindre d'écouter ce qui échappe au sens commun.

- Pour cela, il semble nécessaire de repérer le point de butée qu'une « différence des sexes » conçue rigidement de manière binaire et déshistoricisée présente pour la théorie et la clinique analytiques, pour substituer à ce binarisme la multiplicité propre au genre.

\footnotetext{
${ }^{11}$ Cet article reste programmatique : avant que d'instituer de nouvelles notions pour la métapsychologie, issues de ces savoirs locaux et minoritaires, il convient dans un premier temps de déconstruire certains savoirs hégémoniques. Le propos reste d'éclairer l'historicité irréductible de nombre d'études et de concepts psychanalytiques, pour voir, dans un deuxième temps, comment les resignifier à partir du discours des principaux concernés - mais cela devra faire l'objet de textes à venir.
} 
- On ne peut alors manquer d'analyser les contre-transferts théoriques et cliniques ici impliqués : la «mélancolie de genre» [32] et la norme d'une différence des sexes binaires ont des effets sur chacun/e, et n'épargnent ni l'accueil clinique, ni sa théorisation.

- En outre, il s'agit de renoncer à toute perspective étiologique. Livrer la psychanalyse à une critique psychanalytique revient à déconstruire les postures de «femme» et d' « homme », de « mère » et de « père », de « féminin » et de « masculin », présentées comme catégories psychiques immuables, en pointant leur inscription dans les positions historiques et culturelles des femmes, des hommes et de leur corporalité interprétée.

Mais c'est viser ici une psychanalyse en mouvance, détachée de l'identité à elle même et disposée à se renouveler au gré de chaque transformation de la clinique, une psychanalyse «transidentitaire », qui pourrait reprendre l'injonction de Foucault : «ne me demandez pas qui je suis et ne me dites pas de rester le même : c'est une morale d'état civil ; elle régit nos papiers. Qu'elle nous laisse libre quand il s'agit d'écrire » [33], et, pourrait-on ajouter, d'écouter et de penser $^{12}$.

\section{Références}

1 Derrida J. Etats d'âme de la psychanalyse. Adresse aux Etats Généraux de la psychanalyse. Paris : Galilée ; 2000.

2 Espineira K, Thomas M-Y, Alessandrin A. La Trans-yclopédie. Tout savoir sur les transidentités. Paris : Des Ailes sur un Tracteur ; 2012.

3 Foucault, M. Le corps utopique. Les hétérotopies. Paris : Lignes ; 2009.

4 Stoller R. J. Sex and Gender: The Development of Masculinity and Femininity. New York : Karnac Book ; 1968.

5 Chiland C. Changer de sexe. Paris : Odile Jacob ; 1997.

6 Chiland C. Le transsexualisme. Paris : PUF (Que sais-je? n³671) ; 2003.

7 Chiland C. Problèmes posés par les transsexuels aux psychanalystes. Revue française de psychanalyse. $2005 / 2 ; 69: 563-577$.

\footnotetext{
${ }^{12}$ Cette notion de mouvance et de mutabilité vise, programmatiquement du moins, à détacher la perspective de ce texte de la morale : le propos n'est pas ici de s' «indigner» de certains discours analytiques et de les «dénoncer» (au nom de considérations générales). Il ne s'agit pas d'instituer une «psychanalyse morale», «tolérante », respectueuse de la diversité et bien intentionnée. Ni morale ni immorale, l'approche psychanalytique définie par une constante "différance» de ses identifications, semble devoir, avant de construire, "per via di porre », déconstruire, "per via di levare » [34]. C'est seulement une fois effectuée cette dissolution ou déliaison (« analyse », dans le sens freudien), que l'on peut alors opposer à la généralité des bons sentiments, morale, la singularité d'une posture éthique, en aucun cas universelle mais toujours situationnelle, répondant à la singularité de chaque sujet.
} 
8 Lacan J. Ou Pire. Séminaire, Livre XIX (1971-1972). Paris : Seuil ; 2011.

9 Safouan M. Contribution à la psychanalyse du transsexualisme. In Safouan M. Etudes sur l'Oedipe : introduction à une théorie du sujet. Paris : Seuil ; 1974.

10 Dor J. Structure et perversion. Paris : Denoël ; 1987.

11 Czermak M. Préface. In Frignet H. Sur l'identité sexuelle : à propos du transsexualisme. Paris : Editions de l'Association freudienne ; 1996.

12 Frignet H. Sur l'identité sexuelle : à propos du transsexualisme. Paris : Editions de l'Association freudienne ; 1996.

13 Frignet H. Le transsexualisme. Paris : Déclée de Brower ; 2000.

14 Morel G. Ambiguités sexuelles. Paris : Anthropos ; 2004.

15 Freud S. L'Interprétation des rêves. Paris : PUF ; 1967.

16 Foucault M. Le vrai sexe. Dits et Ecrits IV (1980-1988). Texte 287. Paris :

Gallimard ; 1994.

17 Foucault M. Histoire de la sexualité, Tome I. La volonté de savoir. Paris : Gallimard ; 1976.

18 Foucault M. Le sujet et le pouvoir. Dits et Ecrits IV (1980-1988). Texte 306. Paris : Gallimard ; 1994.

19 Butler J. La vie psychique du pouvoir. Paris : Leo Scheer ; 2002.

20 Butler J. Défaire le Genre. Paris : Amsterdam ; 2012.

21 Bornstein K. Gender-Outlaw. On Men, Women, and the Rest of Us. New York : Vintage ; 1995.

22 Freud S. Trois essais sur le théorie sexuelle. Paris : Gallimard ; 1987.

23 Freud S. Psychogénèse d'un cas d'homosexualité féminin. Névrose, psychose, perversion. Paris : PUF ; 1973.

24 Freud S. Quelques conséquences psychiques de la différence anatomique entre les sexes. La Vie sexuelle. Paris : PUF ; 1969.

25 Schneider M. Surimpressions sexuelles. Cliniques méditerranéennes 2/2006; 74 : 27 42.

26 Laplanche J. Sexual. La sexualité élargie au sens freudien. Paris : PUF ; 2003.

27 Freud S. L'analyse avec fin et l'analyse sans fin. Résultats, idées, problèmes. II. Paris: P.U.F ; 1985.

28 Lacan J. Encore. Le Séminaire, Livre XX (1972-1973). Paris : Seuil ; 1975.

29 Alessandrin A. Le transsexualisme : une catégorie nosographique obsolète. Santé Publique. 2012/3; 24 : 263-268. 
30 Espineira K. Le bouclier thérapeutique : discours et limites d'un appareil de légitimation. Revue internationale de recherche biographique, Le sujet dans la Cité $2011 ; 2: 189-201$.

31 Sironi F. Psychologie(s) des transsexuels et des transgenres. Paris : Odile Jacob ; 2011.

32 Butler J. Troubles dans le genre. Le féminisme et la subversion de l'identité. Paris : La découverte ; 2006.

33 Foucault M. L’archéologie du savoir. Paris : Gallimard, 1969.

34 Freud S. De la Psychothérapie. La technique analytique. Paris : PUF ; 1953. 